\title{
GAPS IN THE PAIRS (BORDER RANK, SYMMETRIC RANK) FOR SYMMETRIC TENSORS
}

\author{
E. BALLICO
}

\begin{abstract}
Fix integers $m \geq 2, s \geq 5$ and $d \geq 2 s+2$. Here we describe the possible symmetric tensor ranks $\leq 2 d+s-7$ of all symmetric tensors (or homogeneous degree $d$ polynomials) in $m+1$ variables with border rank $s$.
\end{abstract}

\section{INTRODUCTION}

An important practical question concerning symmetric tensors (e.g. in Signal Processing, Statistics and Data Analysis) is their " minimal " decomposition as a sum of pure symmetric tensors (see e.g. [11], [16], [6], [10], [15], [4], [14] and references therein). This problem may be translated into the following problem for homogeneous polynomials in $m+1$ variables: for any degree $d$ homogeneous polynomial $f \in \mathbb{K}\left[x_{0}, \ldots, x_{m}\right]$ find the minimal integer $r$ such that $f=\sum_{i=1}^{r} L_{i}{ }^{d}$, where each $L_{i}$ is a homogeneous degree 1 polynomial. The latter problem is translated in the following way into a problem concerning Veronese embeddings of $\mathbb{P}^{m}$.

Let $\nu_{d}: \mathbb{P}^{m} \rightarrow \mathbb{P}^{n_{m, d}}, n_{m, d}:=\left(\begin{array}{c}m+d \\ m\end{array}\right)-1$, denote the degree $d$ Veronese embedding of $\mathbb{P}^{m}$. Set $X_{m, d}:=\nu_{d}\left(\mathbb{P}^{m}\right)$. We often write $n$ instead of $n_{m, d}$. For any subset or closed subscheme $A$ of a projective space $\mathbb{P}^{k}$ let $\langle A\rangle$ denote its linear span. For any integer $s>0$ the $s$-secant variety $\sigma_{s}\left(X_{m, d}\right)$ is the closure in $\mathbb{P}^{n}$ of the union of all linear spaces spanned by $s$ points of $X_{m, d}$. Fix $P \in \mathbb{P}^{n}$. The symmetric rank $\operatorname{sr}(P)$ of $P$ is the minimal cardinality of a finite set $S \subset X_{m, d}$ such that $P \in\langle S\rangle$. The border rank $\operatorname{br}(P)$ of $P$ is the minimal integer $s>0$ such that $P \in \sigma_{s}\left(X_{m, d}\right)$. There is another notion of rank of $P$ (the cactus rank $\operatorname{cr}(P)([7],[5])$, but we do not need to define it, because in the range $\operatorname{br}(P) \leq d+1$ we always have $\operatorname{cr}(P)=b r(P)$ (Remark 1). For any fixed $s \geq 2$ one would like to have the stratification by the

2010 Mathematics Subject Classification. 14N05, 15A69, 15A21.

Key words and phrases. Symmetric tensor rank, border rank, homogeneous polynomial, cactus rank.

The author was partially supported by MIUR and GNSAGA of INdAM (Italy). 
symmetric rank of $\sigma_{s}\left(X_{m, d}\right) \backslash \sigma_{s-1}\left(X_{m, d}\right)$, i.e. to know what are the ranks of the homogeneous degree $d$ polynomials with border rank $s$. This is due to Sylvester if $m=1$, i.e. for binary forms ([9], [15], Theorem 4.1, [4]). For general $m$ this is known if $s=2,3$ ([4]) and if $s=4$ ([1]). For all positive integers $a, b$ set

$$
\sigma_{a, b}\left(X_{m, d}\right):=\left\{P \in \mathbb{P}^{n}: \operatorname{br}(P)=a, \operatorname{sr}(P)=b\right\} .
$$

Notice that $\sigma_{a, b}\left(X_{m, d}\right)=\left\{P \in \sigma_{a}\left(X_{m, d}\right) \backslash \sigma_{a-1}\left(X_{m, d}\right): \operatorname{sr}(P)=b\right\}$ if $a \geq 2$, that $\sigma_{a, a}\left(X_{m, d}\right)$ contains a non-empty open subset of $\sigma_{a}\left(X_{m, d}\right)$ if $\sigma_{a-1}\left(X_{m, d}\right) \neq \mathbb{P}^{n}$ and that $\sigma_{a, b}\left(X_{m, d}\right)=\emptyset$ if $b<a$. If either $m=1$ and $s$ is very low $(s=2,3$ in [4], $s=4$ in [1]), then for fixed $s$ and large $d$ near $s$ several integers are not the symmetric rank of any $P \in \sigma_{s}\left(X_{m, d}\right) \backslash$ $\sigma_{s-1}\left(X_{m, d}\right)$. Here we show that this is the case for arbitrary $s, d$ not too small, but for low ranks, i.e. if we assume $r \leq 2 d+s-7$. We prove the following result.

Theorem 1. Fix integers $m, s, d, r$ such that $m \geq 2, s \geq 5, d \geq 2 s+2$ and $s \leq r \leq 2 d+s-7$.

Then $\sigma_{s, r}\left(X_{m, d}\right) \neq \emptyset$ if and only if one of the following conditions is satisfied:

- $r=s$

- $d+2-s \leq r \leq d+s-2$ and $r+s \equiv d(\bmod 2)$;

- $2 d+2-s \leq r \leq 2 d+s-7$.

If $\sigma_{s}\left(X_{m, d}\right) \supsetneq \sigma_{s-1}\left(X_{m, d}\right)$, then a general $P \in \sigma_{s}\left(X_{m, d}\right)$ satisfies $b r(P)=$ $s=\operatorname{sr}(P)$. Hence in the set-up of Theorem 1 only the pairs $(s, r)$ with $2 \leq s<r$ need to be checked. The statement of Theorem 1 is of the form " if and only if". However, the proofs of both implications use similar tools. The key technical tool used in almost all our lemmas is an inductive method to handle cohomology groups (vanishing and non-vanishing) often called the Horace Method. The starting observation is that for every $s$ as in Theorem 1 there is a zero-dimensional scheme $A \subset \mathbb{P}^{m}$ such that $\operatorname{deg}(A)=s$ and $P \in\left\langle\nu_{d}(A)\right\rangle$ (Remark 1). Moreover if $\operatorname{sr}(P)>\operatorname{br}(P)$, then there is a finite set $B \subset \mathbb{P}^{m}$ such that $\sharp(B)=\operatorname{sr}(P), P \in\left\langle\nu_{d}(A)\right\rangle \cap\left\langle\nu_{d}(B)\right\rangle$ and the scheme $A \cup B$ has two strong properties: $h^{1}\left(\mathcal{I}_{A \cup B}(d)\right)>0$ and there is a line or a conic (say $D)$ such that $\operatorname{deg}((A \cup B) \cap D) \geq \operatorname{deg}(D) \cdot d+2$ and $B \backslash B \cap D=A \backslash A \cap D$ (Lemmas 1 and 5 ). In this way it is easy to get the nonexistence part of Theorem 1 . The existence part in the range $s+r \leq d+2$ (i.e. with $r=d+2-s)$ is done taking $A$ and $B$ contained in a line $L$. To cover the case $r+s \equiv d(\bmod 2)$ and $d+2-s<r \leq d+s-2$ we take $A=(A \cap L) \sqcup E$ and $B=(B \cap L) \sqcup E$ with $E \subset \mathbb{P}^{m} \backslash L, \sharp(E)=(s+r-d-2) / 2, B \cap A \cap L=\emptyset$ and $\operatorname{deg}(A \cap L)+\sharp(B \cap L)=d+2$. To cover the case in which $r+s$ is even and $2 d+2 \leq r+s$ we use a smooth conic $C \subset \mathbb{P}^{m}$ and take $A=(A \cap C) \sqcup E$, 
$B=(B \cap C) \sqcup E$ with $A \cap B \cap C=\emptyset, \operatorname{deg}(A \cap C)+\sharp(B \cap C)=2 d+2$ and $\sharp(E)=(r+s-2 d-2) / 2$. To cover the case $2 d+3-s \leq r \leq 2 d+s-7$ and $r+s$ odd we use a reducible conic instead of $C$; we need two different constructions according to the parity of the integer $(2 d+3+s-r) / 2$ (Lemmas 8 and 9$)$.

In all cases the delicate part is the proof that there is no finite set $S \subset \mathbb{P}^{m}$ such that $P \in\left\langle\nu_{d}(S)\right\rangle$ and $\sharp(S)<\sharp(B)=r$. In all cases we again use Lemmas 1 and 5 .

We work over an algebraically closed base field $\mathbb{K}$ such that $\operatorname{char}(\mathbb{K})=0$.

\section{The PROOF}

For any sheaf $\mathcal{F}$ on $\mathbb{P}^{m}$ and any integer $i \geq 0 \operatorname{set} h^{i}(\mathcal{F}):=\operatorname{dim}\left(H^{i}\left(\mathbb{P}^{m}, \mathcal{F}\right)\right)$. For any scheme $X$, any effective Cartier divisor $D$ of $X$ and any closed subscheme $Z \subset X$ let $\operatorname{Res}_{D}(Z)$ denote the residual scheme of $Z$ with respect to $D$, i.e. the closed subscheme of $X$ with $\mathcal{I}_{Z}: \mathcal{I}_{D}$ as its ideal sheaf. For any $R \in \operatorname{Pic}(X)$ we have the following exact sequence of coherent sheaves (called the residual exact sequence):

$$
0 \rightarrow \mathcal{I}_{\operatorname{Res}_{D}(Z)} \otimes R(-D) \rightarrow \mathcal{I}_{Z} \otimes R \rightarrow \mathcal{I}_{D \cap Z, D} \otimes(R \mid D) \rightarrow 0
$$

We need the following lemma (see [12] for the case in which the scheme $Z$ is reduced, [4], Lemma 34, for the case $z \leq 2 d+1$, and [13] for a strong tool to prove much more in $\mathbb{P}^{2}$ ).

Fix positive integers $m, d$, any $P \in \mathbb{P}^{n_{m, d}}$ and any finite set $B \subset \mathbb{P}^{m}$. We say that $B$ evinces $\operatorname{sr}(P)$ if $P \in\left\langle\nu_{d}(B)\right\rangle$ and $\sharp(B)=\operatorname{sr}(P)$.

Lemma 1. Fix integers $m, d, z$ such that $m \geq 2$ and $0<z<3 d$. Let $Z \subset \mathbb{P}^{m}$ be a zero-dimensional scheme such that $\operatorname{deg}(Z)=z$. If $m>2$, then assume $\operatorname{deg}(Z)-\operatorname{deg}\left(Z_{\text {red }}\right) \leq d$. We have $h^{1}\left(\mathcal{I}_{Z}(d)\right)>0$ if and only if either there is a line $L \subset \mathbb{P}^{m}$ such that $\operatorname{deg}(L \cap Z) \geq d+2$ or there is a conic $T \subset \mathbb{P}^{m}$ such that $\operatorname{deg}(T \cap Z) \geq 2 d+2$.

Proof. Since $Z$ is zero-dimensional, the restriction map $H^{0}\left(Z, \mathcal{O}_{Z}(d)\right) \rightarrow$ $H^{0}\left(W, \mathcal{O}_{W}(d)\right)$ is surjective for any $W \subseteq Z$. Hence for any $W \subseteq Z$ we have $h^{1}\left(\mathcal{I}_{W}(d)\right) \leq h^{1}\left(\mathcal{I}_{Z}(d)\right)$. Since $h^{0}\left(L, \mathcal{O}_{L}(d)\right)=d+1$ for any line $L$ and $h^{0}\left(T, \mathcal{O}_{T}(d)\right)=2 d+1$ for any conic $T$, we get the "if " part. Now assume $h^{1}\left(\mathcal{I}_{Z}(d)\right)>0$.

(a) First assume $m=2$. Apply [13], Remarques (i) at page 116 .

(b) Now assume $m \geq 3$. We use induction on $m$. Let $H_{1} \subset \mathbb{P}^{m}$ be a hyperplane such that $\operatorname{deg}\left(H_{1} \cap Z\right)$ is maximal. Set $Z_{0}:=Z, Z_{1}:=\operatorname{Res}_{H_{1}}\left(Z_{0}\right)$ and $w_{1}:=\operatorname{deg}\left(Z_{0} \cap H_{1}\right)$. As in the proof of [1], Proposition 12, we define recursively the hyperplanes $H_{i} \subset \mathbb{P}^{m}, i \geq 2$, the schemes $Z_{i} \subseteq Z_{i-1}$, and the integers $w_{i}, i \geq 1$, in the following way. Let $H_{i}$ be any hyperplane such that 
$\operatorname{deg}\left(Z_{i-1} \cap H_{i}\right)$ is maximal. Set $Z_{i}:=\operatorname{Res}_{H_{i}}\left(Z_{i-1}\right)$ and $w_{i}:=\operatorname{deg}\left(H_{i} \cap Z_{i-1}\right)$. Any zero-dimensional scheme $F \subset \mathbb{P}^{m}$ with $\operatorname{deg}(F) \leq m$ is contained in a hyperplane. Hence if $w_{i} \leq m-1$, then $w_{i+1}=0$ and $Z_{i}=\emptyset$. Since $z<3 d$, we get $w_{i}=0$ for all $i \geq d$ and $Z_{d}=\emptyset$. For any integer $i>0$ the residual sequence (1) gives the following exact sequence:

$$
0 \rightarrow \mathcal{I}_{Z_{i}}(d-i) \rightarrow \mathcal{I}_{Z_{i-1}}(d-i+1) \rightarrow \mathcal{I}_{H_{i} \cap Z_{i-1}, H_{i}}(d-i+1) \rightarrow 0
$$

Since $h^{1}\left(\mathcal{I}_{Z}(d)\right)>0$ and $Z_{d}=\emptyset,(2)$ gives the existence of integer $x$ such that $1 \leq x \leq d-1$ and $h^{1}\left(H_{x}, \mathcal{I}_{H_{x} \cap Z_{x-1}, H_{x}}(d-x+1)\right)>0$. We call $e$ the minimal such an integer $x$. First assume $e=1$, i.e. assume $h^{1}\left(H_{1}, \mathcal{I}_{Z \cap H_{1}}(d)\right)>0$. Since $\operatorname{deg}\left(Z \cap H_{1}\right) \leq \operatorname{deg}(Z)<3 d$, the inductive assumption on $m$ gives that either there is a line $L \subset H_{1}$ such that $\operatorname{deg}(L \cap Z) \geq 2$ or there is a conic $T \subset H_{1}$ such that $\operatorname{deg}(T \cap Z) \geq 2 d+2$. From now on we assume $e \geq 2$. First assume $w_{e} \geq 2(d-e+1)+2$. Since $w_{i} \geq w_{e}$ for all $i<e$, we get $z \geq 2 e(d-e+1)+2 e$. Since $2 \leq e \leq d-1$ and $z<3 d$, we get a contradiction. Hence $w_{e} \leq 2(d-e+1)+1$. Since $h^{1}\left(H_{e}, \mathcal{I}_{H_{e} \cap Z_{e-1}, H_{e}}(d-e+1)\right)>0$ and $w_{e} \leq 2(d-e+1)+1$, there is a line $L \subset H_{e}$ such that $\operatorname{deg}\left(L \cap Z_{e-1}\right) \geq$ $d-e+3$ ([4], Lemma 34). Since $Z_{e-1} \neq \emptyset, Z_{e-2}$ spans $\mathbb{P}^{m}$. Hence there is a hyperplane $M \subset \mathbb{P}^{m}$ such that $M \supset L$ and $\operatorname{deg}\left(M \cap Z_{e-2}\right) \geq \operatorname{deg}\left(Z_{e-2} \cap\right.$ $L)+m-2 \geq d-e+m+1$. Hence $w_{i} \geq d-e+m+1$ for all $i<e$. Hence $z \geq e(d-e+3)+(e-1)(m-2)$.

First assume $e \geq 3$. Since $3 d>z \geq e(d-e+3)+(e-1)(m-2)$ and $3 \leq e \leq d$, we get a contradiction.

Now assume $e=2$. We have $\operatorname{deg}(L \cap Z) \geq d+1$. If $\operatorname{deg}(L \cap Z) \geq d+2$, then we are done. Hence we may assume $\operatorname{deg}(L \cap Z)=d+1$. Set $W_{0}:=Z$. Let $M_{1} \subset \mathbb{P}^{m}$ be a hyperplane containing $L$ and with $m_{1}:=\operatorname{deg}\left(M_{1} \cap W_{0}\right)$ maximal among the hyperplanes containing $L$. We define recursively the hyperplanes $M_{i} \subset \mathbb{P}^{m}, i \geq 2$, the schemes $W_{i} \subseteq W_{i-1}$, and the integers $m_{i}$, $i \geq 1$, in the following way. Let $M_{i}$ be any hyperplane such that $\operatorname{deg}\left(W_{i-1} \cap\right.$ $\left.M_{i}\right)$ is maximal. Set $W_{i}:=\operatorname{Res}_{M_{i}}\left(W_{i-1}\right)$ and $m_{i}:=\operatorname{deg}\left(H_{i} \cap W_{i-1}\right)$. We have $m_{i} \leq m_{i-1}$ for all $i, m_{1} \geq \operatorname{deg}(L \cap Z)+m-2 \geq d+m-2$ and $m_{i}=0$ if $m_{i-1} \leq m-1$. As above there is a minimal integer $f$ such $1 \leq f \leq d-1$ and $h^{1}\left(M_{f}, \mathcal{I}_{M_{f} \cap W_{f-1}, M_{f}}(d-f+1)\right)>0$. As above we get a contradiction, unless $f=2$. Assume $f=2$. Since $m_{2} \leq z / 2 \leq 2(d-1)+1$, there is a line $D \subset M_{2}$ such that $\operatorname{deg}\left(D \cap W_{1}\right) \geq d+1$. Let $E$ be any connected component of $Z$. If $E$ is reduced, then $L \cap \operatorname{Res}_{M_{1}}(E)=\emptyset$, because $M_{1} \supset L$. If $E$ is not reduced, then $\operatorname{deg}\left(M_{1} \cap \operatorname{Res}_{M_{1}}(E)\right) \leq \operatorname{deg}\left(E \cap M_{1}\right)$, because $\operatorname{Res}_{M_{1}}(E) \subseteq E$. Since $\operatorname{deg}(Z)-\operatorname{deg}\left(Z_{\text {red }}\right) \leq d$, we get $D \neq L$. Assume for the moment that either $D \cap L \neq \emptyset$ or $m \geq 4$, i.e. assume the existence of a hyperplane of $\mathbb{P}^{m}$ containing $D \cup L$. Hence $w_{1} \geq 2 d+1$. Hence $\operatorname{deg}\left(Z_{1}\right) \leq \operatorname{deg}\left(W_{1}\right) \leq d$. Hence $h^{1}\left(\mathcal{I}_{Z_{1}}(d-1)\right)=0$. Hence $h^{1}\left(H_{2}, \mathcal{I}_{Z_{1} \cap H_{2}}(d-1)\right)=0$, contradicting 
the assumption $e=2$. Now assume $m=3$ and $D \cap L=\emptyset$. We may also assume $\operatorname{deg}(L \cap Z)=\operatorname{deg}(D \cap Z)=d+1$. Let $N \subset \mathbb{P}^{3}$ be a general quadric surface containing $D \cup L$. The quadric surface $N$ is smooth. Since $\operatorname{deg}\left(\operatorname{Res}_{N}(Z)\right) \leq z-2 d-2 \leq d-1$, we have $h^{1}\left(\mathcal{I}_{\operatorname{Res}_{N}(Z)}(d-2)\right)=0$. Hence the exact sequence

$$
0 \rightarrow \mathcal{I}_{\operatorname{Res}_{N}(Z)}(d-2) \rightarrow \mathcal{I}_{Z}(d) \rightarrow \mathcal{I}_{Z \cap N, N}(d) \rightarrow 0
$$

gives $h^{1}\left(N, \mathcal{I}_{Z \cap N, N}(d)\right)>0$. Since $D \cap L=\emptyset, D$ and $L$ belong to the same ruling of $N$, say $D, L \in\left|\mathcal{O}_{N}(1,0)\right|$. Since $\operatorname{deg}(Z \cap L)=\operatorname{deg}(Z \cap$ $D)=d+1$, we have $h^{i}\left(N, \mathcal{I}_{Z \cap N, N}(d, d)\right)=h^{i}\left(N, \mathcal{I}_{\operatorname{Res}_{D \cup L}(Z \cap N), N}(d-2, d)\right)$, $i=0,1$. Since $\operatorname{deg}\left(\operatorname{Res}_{D \cup L}(Z \cap N)\right)=\operatorname{deg}(Z \cap N)-2 d-2 \leq d-1$, we have $h^{1}\left(N, \mathcal{I}_{\operatorname{Res}_{D \cup L}(Z \cap N), N}(d-2, d)\right)=0$, a contradiction.

We recall the following result ([3], Lemma 1).

Lemma 2. Fix $P \in \mathbb{P}^{n}$. Assume the existence of zero-dimensional schemes $A, B \subset \mathbb{P}^{m}$ such that $A \neq B, P \in\left\langle\nu_{d}(A)\right\rangle \cap\left\langle\nu_{d}(B)\right\rangle, P \notin\left\langle\nu_{d}\left(A^{\prime}\right)\right\rangle$ for any $A^{\prime} \subsetneq A$ and $P \notin\left\langle\nu_{d}\left(B^{\prime}\right)\right\rangle$ for any $B^{\prime} \subsetneq B$. Then $h^{1}\left(\mathcal{I}_{A \cup B}(d)\right)>0$.

Remark 1. Fix integers $m \geq 1, d \geq 2$ and $P \in \mathbb{P}^{n}$ such that $\operatorname{br}(P) \leq$ $d+1$. By [8], Lemma 2.1.5 and Lemma 2.4.4, there is a smoothable zerodimensional and Gorenstein scheme $A \subset \mathbb{P}^{m}$ such that $\operatorname{deg}(A)=\operatorname{br}(P)$, $P \in\left\langle\nu_{d}(A)\right\rangle$ and $P \notin\left\langle\nu_{d}\left(A^{\prime}\right)\right\rangle$ for any $A^{\prime} \subsetneq A$. We will say that $A$ evinces $\operatorname{br}(P)$. In this range the smoothable rank and the border rank coincide. Now assume $\operatorname{br}(P) \leq(d+1) / 2$. Using Lemma 2 and the inequality $2 s \leq d+1$ we get that $A$ is the unique zero-dimensional scheme $E \subset \mathbb{P}^{m}$ such that $P \in$ $\left\langle\nu_{d}(E)\right\rangle$ and $\operatorname{deg}(E) \leq s$. The uniqueness of $A$ implies that $A$ also evinces the cactus rank $\operatorname{cr}(P)$ of $P$. In particular $\operatorname{cr}(P)=\operatorname{br}(P)$ if $\operatorname{br}(P) \leq(d+1) / 2$.

Lemma 3. Fix a proper linear subspace $L$ of $\mathbb{P}^{m}$, an integer $d \geq 2$ and a finite set $E \subset \mathbb{P}^{m} \backslash L$ such that $\sharp(E) \leq d$. Then $\operatorname{dim}\left(\left\langle\nu_{d}(E \cup L)\right\rangle\right)=$ $\operatorname{dim}\left(\left\langle\nu_{d}(L)\right\rangle\right)+\sharp(E)$. For any closed subscheme $U \subseteq L$ we have $\left\langle\nu_{d}(U \cup\right.$ $E)\rangle \cap\left\langle\nu_{d}(L)\right\rangle=\left\langle\nu_{d}(U)\right\rangle$. For any $O \in\left\langle\nu_{d}(L \cup E)\right\rangle \backslash\left\langle\nu_{d}(E)\right\rangle$, the set $\langle\{O\} \cup$ $\left.\nu_{d}(E)\right\rangle \cap\left\langle\nu_{d}(L)\right\rangle$ is a unique point.

Proof. Since $E$ is a finite set and $E \cap L=\emptyset$, a general hyperplane $H$ containing $L$ contains no point of $E$. Since $E \cap H=\emptyset$, we have $\mathcal{I}_{E \cup H}(d) \cong$ $\mathcal{I}_{E}(d-1)$. Since $\sharp(E) \leq d$, we have $h^{1}\left(\mathcal{I}_{E}(d-1)\right)=0$. Hence $\operatorname{dim}\left(\left\langle\nu_{d}(H \cup\right.\right.$ $E)\rangle)=\operatorname{dim}\left(\left\langle\nu_{d}(H)\right\rangle\right)+\sharp(E)$. Since $L \subseteq H$, we get $\operatorname{dim}\left(\left\langle\nu_{d}(E \cup L)\right\rangle\right)=$ $\operatorname{dim}\left(\left\langle\nu_{d}(L)\right\rangle\right)+\sharp(E)$. Grassmann's formula give $\left\langle\nu_{d}(L)\right\rangle \cap\left\langle\nu_{d}(E)\right\rangle=\emptyset$. Hence $\left\langle\nu_{d}(U \cup E)\right\rangle \cap\left\langle\nu_{d}(L)\right\rangle=\left\langle\nu_{d}(U)\right\rangle$ for any $U \subseteq L$. Fix any $O \in\left\langle\nu_{d}(L \cup E)\right\rangle \backslash$ $\left\langle\nu_{d}(E)\right\rangle$. Since $O \notin\left\langle\nu_{d}(E)\right\rangle$, we have $\operatorname{dim}\left(\left\langle\{O\} \cup \nu_{d}(E)\right\rangle\right)=\operatorname{dim}\left(\left\langle\nu_{d}(E)\right)+1\right.$. Since $O \in\left\langle\nu_{d}(L \cup E)\right\rangle$ and $\left\langle\nu_{d}(L)\right\rangle \cap\left\langle\nu_{d}(E)\right\rangle=\emptyset$, Grassmann's formula gives that $\left\langle\{O\} \cup \nu_{d}(E)\right\rangle \cap\left\langle\nu_{d}(L)\right\rangle$ is a unique point. 
In the same way we get the following result.

Lemma 4. Fix a conic $T \subset \mathbb{P}^{m}$, an integer $d \geq 5$ and a finite set $E \subset \mathbb{P}^{m} \backslash T$ such that $\sharp(E) \leq d-1$. Then $\operatorname{dim}\left(\left\langle\nu_{d}(E \cup T)\right\rangle\right)=\operatorname{dim}\left(\left\langle\nu_{d}(T)\right\rangle\right)+\sharp(E)$. For any closed subscheme $U \subseteq T$ we have $\left\langle\nu_{d}(U \cup E)\right\rangle \cap\left\langle\nu_{d}(T)\right\rangle=\left\langle\nu_{d}(U)\right\rangle$. For any $O \in\left\langle\nu_{d}(T \cup E)\right\rangle \backslash\left\langle\nu_{d}(E)\right\rangle$, the set $\left\langle\{O\} \cup \nu_{d}(E)\right\rangle \cap\left\langle\nu_{d}(T)\right\rangle$ is a unique point.

The following lemma was proved (with $D$ a hyperplane) in [2], Lemma 8. The same proof works for an arbitrary hypersurface $D$ of $\mathbb{P}^{m}$ (see also Remark 2 below).

Lemma 5. Fix $P \in \mathbb{P}^{n}$. Assume the existence of zero-dimensional schemes $A, B \subset \mathbb{P}^{m}$ such that $A \neq B, P \in\left\langle\nu_{d}(A)\right\rangle \cap\left\langle\nu_{d}(B)\right\rangle, P \notin\left\langle\nu_{d}\left(A^{\prime}\right)\right\rangle$ for any $A^{\prime} \subsetneq A$ and $P \notin\left\langle\nu_{d}\left(B^{\prime}\right)\right\rangle$ for any $B^{\prime} \subsetneq B$. Assume that $B$ is reduced. Assume the existence of a positive integer $t \leq d$ and of a degree $t$ hypersurface $D \subset \mathbb{P}^{m}$ such that $h^{1}\left(\mathcal{I}_{\operatorname{Res}_{D}(A \cup B)}(d-t)\right)=0$. Set $E:=B \backslash B \cap D$. Then $\nu_{d}(E)$ is linearly independent, $E=\operatorname{Res}_{D}(A)$ and every unreduced connected component of $A$ is contained in $D$. The linear space $\left\langle\nu_{d}(A)\right\rangle \cap\left\langle\nu_{d}(B)\right\rangle$ is the linear span of its supplementary subspaces $\left\langle\nu_{d}(E)\right\rangle$ and $\left\langle\nu_{d}(A \cap D)\right\rangle \cap\left\langle\nu_{d}(B \cap D)\right\rangle$.

Remark 2. Take the set-up of Lemma 5.

Claim : We have $\left\langle\nu_{d}(A \cap D)\right\rangle \cap\left\langle\nu_{d}(B \cap D)\right\rangle \neq \emptyset$ and there is $Q \in$ $\left\langle\nu_{d}(A \cap D)\right\rangle \cap\left\langle\nu_{d}(B \cap D)\right\rangle$ such that $P \in\left\langle\{Q\} \cup \nu_{d}(E)\right\rangle$.

Proof of the Claim : Lemma 2 gives $h^{1}\left(\mathcal{I}_{A \cup B}(d)\right)>0$. Since $P \notin$ $\left\langle\nu_{d}\left(A^{\prime}\right)\right\rangle$ for any $A^{\prime} \subsetneq A$ and $P \notin\left\langle\nu_{d}\left(B^{\prime}\right)\right\rangle$ for any $B^{\prime} \subsetneq B$, we get $E \neq A$ and $E \neq B$, i.e. $A \cap D \neq \emptyset$ and $B \cap D \neq \emptyset$. The residual exact sequence (1) gives the following exact sequence:

$$
0 \rightarrow \mathcal{I}_{\operatorname{Res}_{D}(A \cup B)}(d-t) \rightarrow \mathcal{I}_{A \cup B}(d) \rightarrow \mathcal{I}_{(A \cup B) \cap, D, D}(d) \rightarrow 0
$$

From (4) and the definition of $E$ we get the last assertion of Lemma 5. Since $P \in\left\langle\nu_{d}(A)\right\rangle \cap\left\langle\nu_{d}(B)\right\rangle, P \notin\left\langle\nu_{d}\left(A^{\prime}\right)\right\rangle$ for any $A^{\prime} \subsetneq A$ and $P \notin\left\langle\nu_{d}\left(B^{\prime}\right)\right\rangle$ for any $B^{\prime} \subsetneq B, \nu_{d}(A)$ and $\nu_{d}(B)$ are linearly independent. Since $h^{1}\left(\mathcal{I}_{A \cup B}(d)\right)>0$ (Lemma 2), the exact sequence (4) gives $h^{1}\left(D, \mathcal{I}_{(A \cup B) \cap, D, D}(d)\right)>0$. Hence the linear independence of $\nu_{d}(A)$ and $\nu_{d}(B)$ implies $\left\langle\nu_{d}(A \cap D)\right\rangle \cap\left\langle\nu_{d}(B \cap\right.$ $D)\rangle \neq \emptyset$. Since $\nu_{d}(A)$ is linearly independent, $P \in\left\langle\nu_{d}(A)\right\rangle \cap\left\langle\nu_{d}(B)\right\rangle, P \notin$ $\left\langle\nu_{d}\left(A^{\prime}\right)\right\rangle$ for any $A^{\prime} \subsetneq A$ and $P \notin\left\langle\nu_{d}\left(B^{\prime}\right)\right\rangle$ for any $B^{\prime} \subsetneq B$, the sets $\langle\{P\} \cup$ $\left.\nu_{d}(E)\right\rangle \cap\left\langle\nu_{d}(A \cap D)\right\rangle$ and $\left\langle\{P\} \cup \nu_{d}(E)\right\rangle \cap\left\langle\nu_{d}(A \cap D)\right\rangle$ are given by a unique point. Call it $Q_{A}$ and $Q_{B}$, respectively. Obviously $P \in\left\langle\nu_{d}(E) \cup\left\{Q_{A}\right\}\right\rangle \cap$ $\left\langle\nu_{d}(E) \cup\left\{Q_{B}\right\}\right\rangle$. Since $P \notin\left\langle\nu_{d}(E)\right\rangle$, we get $Q_{A}=Q_{B}$. Set $Q:=Q_{A}$.

For any reduced projective set $Y \subset \mathbb{P}^{r}$ spanning $\mathbb{P}^{r}$ and any $P \in \mathbb{P}^{r}$ let $r_{Y}(P)$ denote the minimal cardinality of a finite set $S \subset Y$ such that $P \in\langle S\rangle$. The positive integer $r_{Y}(P)$ is often called the $Y$-rank of $P$. 
Lemma 6. Assume $m=2$. Fix integers $w \geq 3$ and $d \geq 4 w-1$. Take lines $L_{1}, L_{2} \subset \mathbb{P}^{2}$ such that $L_{1} \neq L_{2}$. Set $\{O\}:=L_{1} \cap L_{2}$. Let $A_{1} \subset L_{1}$ be the degree $w$ effective divisor of $L_{1}$ with $O$ as its support. Fix $O_{2} \in L_{2} \backslash\{O\}$ and let $A_{2} \subset L_{2}$ the degree $w$ effective divisor of $L_{2}$ with $O_{2}$ as its reduction. Set $A:=A_{1} \cup A_{2}$. Fix $P \in\left\langle\nu_{d}(A)\right\rangle$ such that $P \notin\left\langle\nu_{d}\left(A^{\prime}\right)\right\rangle$ for any $A^{\prime} \subsetneq A$. Then $\operatorname{br}(P)=2 w$ and $\operatorname{sr}(P) \geq 2 d+3-2 w$. There is $P$ as above with $\operatorname{sr}(P)=2 d+3-2 w$.

Proof. Since $\operatorname{deg}(A)=2 w \leq(d+1) / 2$ and $P \notin\left\langle\nu_{d}\left(A^{\prime}\right)\right\rangle$ for any $A^{\prime} \subsetneq A, A$ is the only scheme evincing $b r(P)$ (Remark 1 ).

(a) In this step we prove the existence of $P$ as above and with $\operatorname{sr}(P) \leq$ $2 d+3-2 w$. Fix $B_{1} \subset L_{1} \backslash\{O\}$ such that $\sharp\left(B_{1}\right)=d-w+2$ and $B_{2} \subset L_{2} \backslash\left\{P_{2}\right\}$ such that $\sharp\left(B_{2}\right)=d-w+1$. Since $O \notin B_{1}, \operatorname{deg}\left(A_{1} \cup B_{1}\right)=d+2$ and $\nu_{d}\left(L_{1}\right)$ is a degree $d$ rational normal curve in its linear span, the set $\left\langle\nu_{d}\left(A_{1}\right)\right\rangle \cap\left\langle\nu_{d}\left(B_{1}\right)\right\rangle$ is a single point (call it $P^{\prime}$ ). We have $P^{\prime} \notin\langle W\rangle$ if either $W \subsetneq A_{1}$ or $W \subsetneq B_{1}$. Since $\operatorname{dim}\left(\left\langle\nu_{d}\left(L_{1} \cup L_{2}\right)\right\rangle\right)=2 d, \nu_{d}\left(A_{1} \cup A_{2} \cup B_{1} \cup B_{2}\right)$ spans $\left\langle\nu_{d}\left(L_{1} \cup L_{2}\right)\right\rangle$ and $\operatorname{dim}\left(\left\langle\nu_{d}\left(B_{1} \cup B_{2}\right)\right\rangle\right)=2 d+2-2 w$, the set $\left\langle\nu_{d}\left(A_{1} \cup A_{2}\right)\right\rangle \cap\left\langle\nu_{d}\left(B_{1} \cup B_{2}\right)\right\rangle$ is a line, $M$. Obviously $P^{\prime} \in M$. Take as $P$ any of the points of $M \backslash\left\{P^{\prime}\right\}$. Since $P \in\left\langle\nu_{d}\left(B_{1} \cup B_{2}\right)\right\rangle$, we have $\operatorname{sr}(P) \leq 2 d+3-2 w$.

(b) To conclude the proof it is sufficient to prove that $\operatorname{sr}(P) \geq 2 d+3-$ $2 w$. Assume $\operatorname{sr}(P) \leq 2 d+2-2 w$ and fix $B \subset \mathbb{P}^{2}$ evincing $\operatorname{sr}(P)$. We have $h^{1}\left(\mathcal{I}_{A \cup B}(d)\right)>0$ (Lemma 2) and $\operatorname{deg}(A \cup B) \leq 2 d+2$. Hence either there is a line $D \subset \mathbb{P}^{2}$ such that $\operatorname{deg}(D \cap(A \cup B)) \geq d+2$ or there is a conic $T$ such that $\operatorname{deg}(T \cap(A \cup B)) \geq 2 d+2($ Lemma 1$)$.

(b1) Assume the existence of a line $D$ such that $\operatorname{deg}(D \cap(A \cup B)) \geq$ $d+2$. Since $\operatorname{deg}\left(\operatorname{Res}_{D}(A \cup B)\right) \leq(2 d-2 w+2)+2 w-d-2=d$, we have $h^{1}\left(\mathcal{I}_{\operatorname{Res}_{D}(A \cup B)}(d-1)\right)=0$. Hence Lemma 5 gives $A \subset D$, a contradiction.

(b2) Assume the existence of a conic $T$ such that $\operatorname{deg}(T \cap(A \cup B)) \geq$ $2 d+2$. Since $\operatorname{deg}(A)+\operatorname{deg}(B) \leq 2 d+2$, we get $A \cap B=\emptyset, \sharp(B)=2 d+2-2 w$ and $A \cup B \subset T$. We have $\operatorname{deg}\left(L_{2} \cap A\right)=w+1$ and $\operatorname{deg}\left(L_{1} \cap A\right)=w$. Since $w \geq 3$, the Bezout theorem implies that $L_{1} \cup L_{2}$ is the unique conic containing $A$. Hence $T=L_{1} \cup L_{2}$. Set $B_{i}:=B \cap L_{i}$. First assume $\sharp\left(B_{1}\right) \geq d-w+2$. Since $\sharp\left(B_{1}\right) \leq d-w$, the scheme $\operatorname{Res}_{L_{1}}(A \cup B)=A_{2} \cup B_{2}$ has degree $\leq d$. Hence $h^{1}\left(\mathcal{I}_{\operatorname{Res}_{L_{1}}(A \cup B)}(d-1)\right)=0$. Lemma 5 gives $A \subset L_{1}$, a contradiction. Now assume $\sharp\left(B_{1}\right) \leq d-w+1$. Let $G \subset L_{1}$ be the degree $w-1$ effective divisor of $L_{1}$ with $O$ as its support. Since $\operatorname{Res}_{L_{2}}(A \cup B)=G \cup B_{1}$ has degree $\leq d$, Lemma 5 implies $A \subset L_{2}$, a contradiction.

Lemma 7. Assume $m=2$. Fix integers $w \geq 2$ and $d \geq 4 w+1$. Take lines $L_{1}, L_{2} \subset \mathbb{P}^{2}$ such that $L_{1} \neq L_{2}$. Set $\{O\}:=L_{1} \cap L_{2}$. Let $A_{1} \subset L_{1}$ be the degree $w+1$ effective divisor of $L_{1}$ with $O$ as its support. Fix $O_{2} \in L_{2} \backslash\{O\}$ and let $A_{2} \subset L_{2}$ the degree $w$ effective divisor of $L_{2}$ with $O_{2}$ as its reduction. 
Set $A:=A_{1} \cup A_{2}$. Fix $P \in\left\langle\nu_{d}(A)\right\rangle$ such that $P \notin\left\langle\nu_{d}\left(A^{\prime}\right)\right\rangle$ for any $A^{\prime} \subsetneq A$. Then $\operatorname{br}(P)=2 w+1$ and $\operatorname{sr}(P) \geq 2 d+2-2 w$. There is a $P$ as above with $\operatorname{sr}(P)=2 d+2-2 w$.

Proof. Copy the proof of Lemma 6. In step (b2) we have $T=L_{1} \cup L_{2}$, because $\left.\operatorname{deg}\left(L_{1} \cap A\right)=\operatorname{deg}\left(L_{2} \cap A\right)=w+1 \geq 3\right)$.

Lemma 8. Fix integers $w \geq 3, s \geq 2 w$ and assume $d \geq 2 s-1$. Fix lines $D, R \subset \mathbb{P}^{m}$ such that $D \neq R$ and $D \cap R \neq \emptyset$. Set $\{O\}:=D \cap R$. Let $U$ be the plane spanned by $D \cup R$. Let $E \subset U$ be a general subset with cardinality $s-2 w$. Let $A_{1} \subset D$ be the zero-dimensional degree $w$ subscheme of $D$ with $O$ as its support. Fix $O^{\prime} \in R \backslash\{O\}$ and call $A_{2}$ the zero-dimensional subscheme of $R$ with $O^{\prime}$ as its support and degree $w$. Set $A:=A_{1} \cup A_{2}$. There is $P \in\left\langle\nu_{d}\left(A_{1} \cup A_{2} \cup E\right)\right\rangle$ such that $b r(P)=s$ and $\operatorname{sr}(P)=2 d+3+s-4 w$.

Proof. We will always compute the residual schemes with respect to divisors of $U$. Notice that $A:=A_{1} \cup A_{2} \cup E$ is curvilinear and hence it only has finitely many subschemes. Hence there is $P \in\left\langle\nu_{d}\left(A_{1} \cup A_{2} \cup E\right)\right\rangle$ such that $P \notin\left\langle\nu_{d}(F)\right\rangle$ for any $F \subsetneq A_{1} \cup A_{2} \cup E$. Since $\operatorname{deg}(A)=s \leq(d+1) / 2$, we get $s r(P)=s$ and that $A$ is the only subscheme of $\mathbb{P}^{m}$ evincing $s b(P)$ (Remark $1)$.

(a) Fix $P \in\left\langle\nu_{d}\left(A_{1} \cup A_{2} \cup E\right)\right\rangle$ such that $P \notin\left\langle\nu_{d}(F)\right\rangle$ for any $F \subsetneq$ $A_{1} \cup A_{2} \cup E$. In this step we prove that $\operatorname{sr}(P) \geq 2 d+3+s-2 w$. Assume $s r(P) \leq 2 d+2+s-2 w$. By [9], Proposition 3.1, or [15], subsection 3.2, there is $B \subset U$ evincing $\operatorname{sr}(P)$. Since $A$ is not reduced, we have $A \neq B$. Hence $h^{1}\left(U, \mathcal{I}_{A \cup B}(d)\right)>0$. Set $W:=A \cup B$. Since $\operatorname{deg}(W) \leq 2 d+2 s-6<3 d$, either there is a line $L \subset U$ such that $\operatorname{deg}(L \cap W) \geq d+2$ or there is a conic $T \subset U$ such that $\operatorname{deg}(T \cap W) \geq 2 d+2$ (Lemma 1$)$.

(a1) Assume the existence of a line $L \subset U$ such that $\operatorname{deg}(L \cap W) \geq$ $d+2$. If $h^{1}\left(U, \mathcal{I}_{\operatorname{Res}_{L}(W)}(d-1)\right)=0$, then Lemma 5 gives $A_{1} \cup A_{2} \subset$ $L$, absurd. Hence $h^{1}\left(U, \mathcal{I}_{\operatorname{Res}_{L}(W)}(d-1)\right)>0$. Since $\operatorname{deg}\left(\operatorname{Res}_{L}(W)\right) \leq$ $2(d-1)+1$, there is a line $L^{\prime} \subset U$ such that $\operatorname{deg}\left(L^{\prime} \cap \operatorname{Res}_{L}(W)\right) \geq d+1$. Since $\operatorname{deg}\left(\operatorname{Res}_{L \cup L^{\prime}}(W)\right) \leq d-1$, Lemma 5 gives $A_{1} \cup A_{2} \subset L \cup L^{\prime}$ and $\operatorname{Res}_{L \cup L^{\prime}}(A)=B \backslash B \cap\left(L \cup L^{\prime}\right)$. Since $w \geq 3$, the Bezout theorem gives $L \cup L^{\prime}=D \cup R$. Hence $E \subset B$ and $B \backslash E \subset D \cup R$. Lemma 5 and Remark 2 applied to $D \cup R$ give the existence of $Q \in\left\langle\nu_{d}(A \cap(D \cup R))\right\rangle$ such that $A_{1} \cup A_{2}$ evinces $\operatorname{br}(Q)$, while $B \backslash E$ evinces $\operatorname{sr}(Q)$. Since $\sharp(B \backslash E) \leq 2 d+2-2 w$, either $\sharp((B \backslash E) \cap D) \leq d+1-w$ or $\sharp((B \backslash E) \cap R) \leq d-w$. First assume $\sharp((B \backslash E) \cap D) \leq d+1-w$. Since $\operatorname{deg}\left(\operatorname{Res}_{R}\left(A_{1} \cup A_{2}\right)\right)=w-1$, we have $h^{1}\left(\mathcal{I}_{\operatorname{Res}_{R}((A \cup B) \cap(D \cup R))}(d-1)\right)=0$. Hence Lemma 5 applied to $A \cap(D \cap R)$ and $B \cap(D \cup R)$ gives $\operatorname{Res}_{R}\left(A_{1} \cup A_{2}\right)=\operatorname{Res}_{R}(B \cap(D \cup R))$. Since $B$ is reduced, we get $w \leq 2$, a contradiction. 
(a2) Now we assume the existence of a conic $T \subset U$ such that $\operatorname{deg}(T \cap$ $W) \geq 2 d+2$. Since $\operatorname{deg}\left(\operatorname{Res}_{T}(W)\right) \leq d-1$, we have $h^{1}\left(\mathcal{I}_{\operatorname{Res}_{T}(W)}(d-2)\right)=0$. Hence the case $t=2$ of Lemma 5 gives $A_{1} \cup A_{2} \subset T$ and $B \backslash B \cap T=\operatorname{Res}_{T}(A)$. Since $w \geq 3$, and $A_{1} \cup A_{2} \subset T$, the Bezout theorem gives $T=D \cup R$. We work as in step (b1) (notice that in the case $L \cup L^{\prime}=D \cup R$ we only used that $\left.\operatorname{deg}\left(\left(L \cup L^{\prime}\right) \cap(A \cup B)\right) \geq 2 d+2\right)$.

(b) In this step we check the existence of $P \in\left\langle\nu_{d}\left(A_{1} \cup A_{2} \cup E\right)\right\rangle$ such that $P \notin\left\langle\nu_{d}(F)\right\rangle$ for any $F \subsetneq A_{1} \cup A_{2} \cup E$ and $\operatorname{sr}(P)=2 d+3+s-2 w$ and $\operatorname{br}(P)=s$. Lemma 6 gives the existence of $O \in\left\langle\nu_{d}\left(A_{1} \cup A_{2}\right)\right\rangle$ such that $\operatorname{sr}(O) \leq 2 d+3-2 w$ and $O \notin\left\langle\nu_{d}(G)\right\rangle$ for any $G \subsetneq A_{1} \cup A_{2}$. Take a general $P \in\left\langle\{O\} \cup \nu_{d}(E)\right\rangle$. Obviously $\operatorname{sr}(P) \leq \operatorname{sr}(O)+\sharp(E) \leq 2 d+3+s-2 w$. Step (b) gives $\operatorname{sr}(P)=2 d+3+s-2 w$. Assume $b r(P)<s$. Since $\operatorname{br}(P) \leq d+1$, there is a zero-dimensional scheme $W \subset \mathbb{P}^{m}$ such that $\operatorname{deg}(W)=\operatorname{br}(P), P \in$ $\left\langle\nu_{d}(W)\right\rangle$ and $P \notin\left\langle\nu_{d}\left(W^{\prime}\right)\right\rangle$ for any $W^{\prime} \subsetneq W$. First assume $W \nsubseteq A_{1} \cup A_{2} \cup F$. Lemma 2 gives $h^{1}\left(\mathcal{I}_{A_{1} \cup A_{2} \cup E \cup W}(d)\right)>0$. Since $b s(P)+s \leq 2 d+1$, there is a line $L \subset \mathbb{P}^{m}$ such that $\operatorname{deg}\left(L \cap\left(A_{1} \cup A_{2} \cup E \cup W\right)\right) \geq d+2$. As in step (b1) we get a contradiction. Now assume $W \subsetneq A_{1} \cup A_{2} \cup E$. Set $A^{\prime}:=\left(A_{1} \cup A_{2}\right) \cap W$. Lemma 3 gives $\left\langle\{P\} \cup \nu_{d}(E)\right\rangle \cap\left\langle\nu_{d}\left(A_{1} \cup A_{2}\right)\right\rangle=\{O\}$ and $\left\langle\{P\} \cup \nu_{d}\left(E^{\prime}\right)\right\rangle \cap\left\langle\nu_{d}\left(A_{1} \cup A_{2}\right)\right\rangle$. Since $P \in\left\langle\nu_{d}(W)\right\rangle$ and $P \notin\left\langle\nu_{d}\left(W^{\prime}\right)\right\rangle$ for any $W^{\prime} \subsetneq W$, the set $\left\langle\{P\} \cup\left\langle\nu_{d}(W \cap E)\right\rangle \cap\left\langle\nu_{d}\left(A^{\prime}\right)\right\rangle\right.$, is a single point. Hence $E \subseteq W$ and $\{O\} \in\left\langle\nu_{d}\left(A^{\prime}\right)\right\rangle$, contradicting the choice of $O$.

Quoting Lemma 7 instead of Lemma 6 we get the following result.

Lemma 9. Fix integers $w \geq 2$ and $s \geq 2 w+1$, Assume $d \geq 2 s-1$. Fix lines $D, R \subset \mathbb{P}^{m}$ such that $D \neq R$ and $D \cap R \neq \emptyset$. Set $\{O\}:=D \cap R$. Let $U$ be the plane spanned by $D \cup R$. Let $E \subset U$ be a general subset with cardinality $s-2 w-1$. Let $A_{1} \subset D$ be the zero-dimensional degree $w+1$ subscheme of $D$ with $O$ as its support. Fix $O^{\prime} \in R \backslash\{O\}$ and call $A_{2}$ the zero-dimensional subscheme of $R$ with $O^{\prime}$ as its support and degree $w$. Set $A:=A_{1} \cup A_{2}$. There is $P \in\left\langle\nu_{d}\left(A_{1} \cup A_{2} \cup E\right)\right\rangle$ such that $\operatorname{br}(P)=s$ and $\operatorname{sr}(P)=2 d+1+s-4 w$.

Proof of Theorem 1. Since the cases $s=2,3$ are true by [4], Theorems 32 and 37, we may assume $s \geq 4$ (the case $(s, r)=(3,2 d-1)$ does not occur in the statement of Theorem 1 , because we assumed $r \leq 2 d+s-7$; this inequality is used in steps (d) and (e) below).

Notice that $\sigma_{s}\left(X_{m, d}\right) \backslash \sigma_{s-1}\left(X_{m, d}\right) \neq \emptyset$ (e.g., because $s(m+1)<\left(\begin{array}{c}m+d \\ m\end{array}\right)$ ). Fix $P \in \sigma_{s}\left(X_{m, d}\right) \backslash \sigma_{s-1}\left(X_{m, d}\right)$ and write $r:=s r(P)$. Since $P \notin \sigma_{s-1}\left(X_{m, d}\right)$ we have $r \geq s$. Since $\sigma_{s}\left(X_{m, d}\right) \neq \sigma_{s-1}\left(X_{m, d}\right)$ a non-empty open subset of $\sigma_{s}\left(X_{m, d}\right)$ is formed by points with rank $s$. Hence to prove Theorem 1 we may assume $r>s$. By Remark 1 there is a unique degree $s$ zerodimensional scheme $A \subset \mathbb{P}^{m}$ such that $P \in\left\langle\nu_{d}(A)\right\rangle$ and this scheme is smoothable. By Remark 1 there is no zero-dimensional scheme $A_{1} \subset \mathbb{P}^{m}$ 
such that $\operatorname{deg}\left(A_{1}\right)<s$ and $P \in\left\langle\nu_{d}\left(A_{1}\right)\right\rangle$. Hence $P$ has cactus rank $s$ and $P \notin\left\langle\nu_{d}\left(A^{\prime}\right)\right\rangle$ for any $A^{\prime} \subsetneq A$. Since $\operatorname{sr}(P)=r$ there is a finite set $B \subset \mathbb{P}^{m}$ such that $\sharp(B)=r, P \in\left\langle\nu_{d}(B)\right\rangle$ and $P \notin\left\langle\nu_{d}\left(B^{\prime}\right)\right\rangle$ for any $B^{\prime} \subsetneq B$. Set $W:=A \cup B$. We have $\operatorname{deg}(W) \leq \operatorname{deg}(A)+\operatorname{deg}(B)=r+s$ and equality holds if and only if $A \cap B=\emptyset$. Lemma 2 gives $h^{1}\left(\mathcal{I}_{W}(d)\right)>0$. Hence $\operatorname{deg}(W) \geq d+2$ (e.g., by [4], Lemma 34). Therefore $\sigma_{s, x}\left(X_{m, d}\right)=\emptyset$ if $s+1 \leq x \leq d-s+1$.

We have $\sigma_{s, d-s+2}\left(X_{m, d}\right) \neq \emptyset$, because $\sigma_{s, d-s+2}\left(X_{1, d}\right) \neq \emptyset$ by a theorem of Sylvester ([9], [15], Theorem 4.1, or [4]) and for any line $L \subset \mathbb{P}^{m}$ and any $P \in\left\langle\nu_{d}(L)\right\rangle$ the symmetric rank and the border rank of $P$ are the same with respect to $X_{m, d}$ or with respect to $\nu_{d}(L) \cong X_{1, d}$ ([15], subsection 3.2).

(a) In this step we prove that $\sigma_{s, r}\left(X_{m, d}\right) \neq \emptyset$ for every $r \in\{d-s+$ $3, \ldots, d+s-2\}$ such that $r+s \equiv d(\bmod 2)$. Fix $r \in\{d-s+3, \cdots, d+s-2\}$ such that $r+s \equiv d(\bmod 2)$. Set $b:=(d+2+s-r) / 2$. Since $r+s \equiv d$ $(\bmod 2)$, we have $b \in \mathbb{Z}$. Since $r \leq d+s-2$, we have $b \geq 2$. Since $r \geq d-s+3$, we have $b<s$. Since $d \geq 2 s-2$, we have $r>s$ and hence $2 b<d+2$. Fix a line $L \subset \mathbb{P}^{m}$ and a connected zero-dimensional scheme $Z^{\prime} \subset L$ such that $\operatorname{deg}\left(Z^{\prime}\right)=b$. Take any $Q \in\left\langle\nu_{d}\left(Z^{\prime}\right)\right\rangle$ such that $Q \notin\left\langle\nu_{d}\left(Z^{\prime \prime}\right)\right\rangle$ for any $Z^{\prime \prime} \subsetneq Z^{\prime}$ ( $Q$ exists and the set of all such points $Q$ is a non-empty open subset of a projective space of dimension $\operatorname{deg}\left(Z^{\prime}\right)-1$, because $Z^{\prime}$ is a divisor of the smooth curve $L)$. Fix any set $E \subset \mathbb{P}^{m} \backslash L$ such that $\sharp(E)=s-b=(s+r-d-2) / 2$. Since $E \cap L=\emptyset$, we have $\operatorname{deg}\left(Z^{\prime} \cup E\right)=s$. Since $d \geq s-1$, we have $\operatorname{dim}\left(\left\langle\nu_{d}\left(Z^{\prime} \cup E\right)\right\rangle\right)=s-1$. Since $L$ is contained in a smooth curve, $Z^{\prime}$ is curvilinear. Since $E$ is a finite set, the scheme $Z^{\prime} \cup E$ is curvilinear. We claim that any zero-dimensional curvilinear subscheme $W \subset \mathbb{P}^{m}$ has only finitely many subschemes. Indeed, $W$ is contained in a smooth curve $C$ and hence we may write $W=\sum_{i=1}^{x} a_{i} Q_{i}$ for some $x \in \mathbb{N} \backslash\{0\}, a_{i} \in \mathbb{N} \backslash\{0\}$ and $Q_{i} \in C$. The subschemes of $W$ are the effective divisors of $C$ of the form $\sum_{i=1}^{x} b_{i} Q_{i}$ for some $b_{i} \in\left\{0, \ldots, a_{i}\right\}$. Hence $W$ has exactly $\prod_{i=1}^{x}\left(a_{i}+1\right)$ subschemes. Hence $Z^{\prime} \cup E$ has only finitely many closed subschemes. Fix any $O \in\left\langle\nu_{d}\left(Z^{\prime} \cup E\right)\right\rangle$ such that $O \notin\left\langle\nu_{d}(F)\right\rangle$ for any $F \subsetneq Z^{\prime} \cup E(O$ exists and the set of all such points $O$ is a non-empty open subset of the $(s-1)$ dimensional projective space $\left\langle\nu_{d}\left(Z^{\prime} \cup E\right)\right\rangle$, because $Z^{\prime} \cup E$ has only finitely many subschemes and $O \notin\left\langle\nu_{d}\left(L \cup E^{\prime}\right)\right\rangle$ for any $\left.E^{\prime} \subsetneq E\right)$. Let $A^{\prime} \subset L$ be a set evincing $\operatorname{sr}(Q)$ with respect to the rational normal curve $\nu_{d}(L)$. A theorem of Sylvester gives $\sharp\left(A^{\prime}\right)=d-\operatorname{deg}\left(Z^{\prime}\right)+2([9]$, [15], Theorem 4.1, or [4]). Set $G:=A^{\prime} \cup E$. Since $E \cap L=\emptyset$, we have $\sharp(G)=r$.

Claim 1: $\operatorname{br}(O)=s$. 
Proof of Claim 1: We have $O \in\left\langle\nu_{d}\left(Z^{\prime} \cup E\right)\right\rangle$ and $O \notin\left\langle\nu_{d}(F)\right\rangle$ for any $F \subsetneq Z^{\prime} \cup E$. Apply Remark 1 .

Claim 2: $\operatorname{sr}(O)=r$ and $G$ evinces $\operatorname{sr}(O)$.

Proof of Claim 2: Since $P \in\left\langle\nu_{d}(G)\right\rangle$, we have $\operatorname{sr}(P) \leq r$. Since $s \leq(d+1) / 2, Z^{\prime} \cup E$ is the only scheme evincing $\operatorname{br}(O)$. Since $Z^{\prime}$ is not reduced, we get $\operatorname{sr}(P)>s$. Fix any $U \subset \mathbb{P}^{m}$ evincing $\operatorname{sr}(P)$. Since $s r(P)+b r(P) \leq 2 d+1, \operatorname{sr}(P)>b r(P)$ and $Z^{\prime} \cup E$ evinces $b r(O)$, [3], Theorem 1 , gives the existence of a line $D \subset \mathbb{P}^{m}$ such that $\left(Z^{\prime} \cup E\right) \backslash D \cap\left(Z^{\prime} \cup E\right)=$ $U \backslash U \cap D$ and every unreduced connected component of $Z^{\prime} \cup E$ is contained in $D$. Since $Z^{\prime}$ is not reduced, we get $D=L$. Hence $U \backslash U \cap L=E$. Since $O \in\left\langle\nu_{d}\left(Z^{\prime} \cup E\right)\right\rangle \subseteq\left\langle\nu_{d}(L \cup E)\right\rangle$, Lemma 3 gives that $\left\langle\{O\} \cup \nu_{d}(E)\right\rangle \cap\left\langle\nu_{d}(L)\right\rangle$ is a single point (call it $Q^{\prime}$ ). Since $O \in\left\langle\{Q\} \cup \nu_{d}(E)\right\rangle$ and $Q \in\left\langle\nu_{d}(L)\right\rangle$, we have $Q^{\prime}=Q$. Lemma 3 gives $Q \in\left\langle\nu_{d}(L \cap U)\right\rangle$. Since $A^{\prime}$ evinces $\operatorname{sr}(Q)$, we get $\operatorname{sr}(P)=\sharp(U) \geq \sharp(E)+\sharp\left(A^{\prime}\right)$, concluding the proof of Claim 2 .

The point $O$ shows that $\sigma_{s, r}\left(X_{m, d}\right) \neq \emptyset$ for any $r \in\{d+3-s, \ldots, d+s-2\}$ such that $r+s \equiv d(\bmod 2)$.

(b) Fix any integer $r$ such that $r \in\{d-s+3, \ldots, d+s-2\}$ and $r+s \equiv d+$ $1(\bmod 2)$. In this step we prove that $\sigma_{s, r}\left(X_{m, d}\right)=\emptyset$. Assume the existence of $P \in \sigma_{s, r}\left(X_{m, d}\right)$. Fix $A \subset \mathbb{P}^{m}$ evincing $\operatorname{br}(P)$ and $B \subset \mathbb{P}^{m}$ evincing $\operatorname{sr}(P)$. Since $r>s$ we have $A \neq B$. As in step (a) we get the existence of a line $D \subset \mathbb{P}^{m}$ such that $\operatorname{deg}((A \cup B) \cap D) \geq d+2$, every unreduced connected component of $A$ is contained in $D$ and $\operatorname{Res}_{D}(A)=B \backslash B \cap D$. Set $E:=B \backslash B \cap D$. By Lemma 3 the set $\left\langle\nu_{d}(D)\right\rangle \cap\left\langle\nu_{d}(E) \cup\{P\}\right\rangle$ is a unique point, $O$, and $O \in\left\langle\nu_{d}(A \cap D)\right\rangle \cap\left\langle\nu_{d}(B \cap D)\right\rangle$. Since $\operatorname{deg}(A)=\operatorname{deg}(A \cap D)+\sharp(E)$ (resp. $\sharp(B)=\sharp(B \cap D)+\sharp(E)), A \cap D$ evinces $\operatorname{br}(P)$ (resp. $B \cap D$ evinces $\operatorname{sr}(O))$. The quoted theorem of Sylvester gives $\operatorname{sr}(O)+b r(O)=d+2$. Hence $s+r=2 \cdot \sharp(E)+\operatorname{sr}(O)+b r(O) \equiv d(\bmod 2)$, a contradiction.

(c) In this step we fix an integer $r$ such that $d+s-1 \leq r \leq 2 d+$ $1-s$. In order to obtain a contradiction we assume $\sigma_{s, r}\left(X_{m, d}\right) \neq \emptyset$ and fix $P \in \sigma_{s, r}\left(X_{m, d}\right)$. Take $A \subset \mathbb{P}^{m}$ evincing $\operatorname{br}(P)$ and $B \subset \mathbb{P}^{m}$ evincing $\operatorname{sr}(P)$. Let $A_{1}$ be the union of the connected components of $A$ which are not reduced. Since $r>s$, we have $A_{1} \neq \emptyset$. Hence $\operatorname{deg}\left(A_{1}\right) \geq 2$. Lemma 2 gives $h^{1}\left(\mathcal{I}_{A \cup B}(d)\right)>0$. Since $\operatorname{deg}(A \cup B) \leq s+r \leq 2 d+1$, there is a line $D \subset \mathbb{P}^{m}$ such that $\operatorname{deg}((A \cup B) \cap D) \geq d+2$ (Lemma 1 or [4], Lemma 34). Set $E:=B \backslash B \cap D$. Since $e:=\operatorname{deg}\left(\operatorname{Res}_{D}(A \cup B)\right) \leq r+s-d-2 \leq d$, we have $h^{1}\left(\mathcal{I}_{\operatorname{Res}_{D}(A \cup B)}(d-1)\right)=0$. Hence Lemma 5 gives $A_{1} \subset D$ and $A \backslash A \cap D=E, e=\sharp(E)$ and $e=\operatorname{deg}(A \backslash A \cap D) \leq s-\operatorname{deg}\left(A_{1}\right)$. Since $A$ evinces $\operatorname{br}(P)$, the set $\left\langle\nu_{d}(E) \cup\{P\}\right\rangle \cap\left\langle\nu_{d}\left(A_{1}\right)\right\rangle$ is a unique point, $O$. 
Sylvester's theorem gives $\operatorname{sr}(O) \leq d$. Since $P \in\left\langle\nu_{d}(E) \cup\{O\}\right\rangle$, we get $s r(P) \leq e+d \leq d+s-2$, a contradiction.

(d) In this step we prove that $\sigma_{s, r}\left(X_{m, d}\right) \neq \emptyset$ for every integer $r$ such that $2 d+2-s \leq r \leq 2 d+s-7$ and $r+s \equiv 0(\bmod 2)$. Set $b:=(2 d+2+$ $s-r) / 2$. Since $r+s \equiv 0(\bmod 2)$, we have $b \in \mathbb{Z}$. Since $r \leq 2 d+s-7$, we have $b \geq 9 / 2$ and hence $b \geq 5$. Since $r \geq 2 d+2-s$, we have $b \leq s$. We may assume $m=2\left([15]\right.$, subsection 3.2). Fix a smooth conic $C \subset \mathbb{P}^{2}$, a connected zero-dimensional scheme $A_{1} \subset C$ such that $\operatorname{deg}\left(A_{1}\right)=b$ and a general set $E \subset \mathbb{P}^{2} \backslash C$ such that $\sharp(E)=s-b$. We have $\sharp(E) \leq d-1$. Set $A:=A_{1} \cup E$ and $\left\{O^{\prime}\right\}:=\left(A_{1}\right)_{\text {red }}$. Fix $P \in\left\langle\nu_{d}(A)\right\rangle$ such that $P \notin\left\langle\nu_{d}(F)\right\rangle$ for any scheme $F \subsetneq A\left(P\right.$ exists, because $A$ is curvilinear and $\left.\operatorname{dim}\left(\left\langle\nu_{d}(A)\right\rangle\right)=\operatorname{deg}(A)-1\right)$. Since $s=\operatorname{deg}(A) \leq(d+1) / 2$, then $\operatorname{br}(P)=s$ and $A$ is the only scheme evincing $\operatorname{br}(P)$ (Remark 1). Lemma 4 gives that the set $\langle\{P\} \cup E\rangle \cap\left\langle\nu_{d}(C)\right\rangle$ is a unique point, $O$, that $O \in\left\langle\nu_{d}\left(A_{1}\right)\right\rangle$ and that $\operatorname{sr}(O)=b$. Since $b \leq d+1$, the quoted theorem of Sylvester gives $r_{\nu_{d}(C)}(O)=2 d+2-b$. Fix $B_{1} \subset C$ such that $\nu_{d}\left(B_{1}\right)$ evinces $r_{\nu_{d}(C)}(O)$, i.e. take $B_{1} \subset C$ such that $\sharp\left(B_{1}\right)=2 d+2-b$, $O \in\left\langle\nu_{d}\left(B_{1}\right)\right\rangle$ and $O \notin\left\langle\nu_{d}(F)\right\rangle$ for any $F \subsetneq B_{1}$. We have $P \in\left\langle\nu_{d}\left(B_{1} \cup E\right)\right\rangle$. Lemma 4 also gives $P \notin\left\langle\nu_{d}(G)\right\rangle$ for any $G \subsetneq B_{1} \cup E$. Since $\operatorname{br}(P)=s, P \in$ $\left\langle\nu_{d}\left(B_{1} \cup E\right)\right\rangle$ and $\sharp\left(B_{1} \cup E\right)=r$, to prove that $\sigma_{s, r}\left(X_{m, d}\right) \neq \emptyset$ it is sufficient to prove that $\operatorname{sr}(P) \geq r$. Assume $\operatorname{sr}(P)<r$ and take $B$ evincing $\operatorname{sr}(P)$. We have $h^{1}\left(\mathcal{I}_{A \cup B}(d)\right)>0$ (Lemma 2). Since $\operatorname{deg}(A \cup B) \leq s+r-1<3 d$, either there is a line $D \subset \mathbb{P}^{2}$ such that $\operatorname{deg}(D \cap(A \cup B)) \geq d+2$ or there is a conic $T$ such that $\operatorname{deg}(T \cap(A \cup B)) \geq 2 d+2$ (Lemma 1$)$.

(d1) Here we assume the existence of a line $D \subset \mathbb{P}^{2}$ such that $\operatorname{deg}(D \cap$ $(A \cup B)) \geq d+2$. If $h^{1}\left(\mathcal{I}_{\operatorname{Res}_{D}(A \cup B)}(d-1)\right)=0$, then Lemma 5 gives $A_{1} \subset D$, a contradiction. Hence $h^{1}\left(\mathcal{I}_{\operatorname{Res}_{D}(A \cup B)}(d-1)\right)>0$. Since $\operatorname{deg}\left(\operatorname{Res}_{D}(A \cup\right.$ $B)) \leq r+s-1-d-2 \leq 2(d-1)+1$, Lemma 1 or [4], Lemma 34, give the existence of a line $D^{\prime} \subset \mathbb{P}^{2}$ such that $\operatorname{deg}\left(D^{\prime} \cap \operatorname{Res}_{D}(A \cup B)\right) \geq d+1$. Hence $\operatorname{deg}\left((A \cup B) \cap\left(D \cup D^{\prime}\right)\right) \geq 2 d+3$. Hence $\operatorname{deg}\left(\operatorname{Res}_{D \cup D^{\prime}}(A \cup B)\right) \leq d-1$. Hence $h^{1}\left(\mathcal{I}_{\operatorname{Res}_{D \cup D^{\prime}}(A \cup B)}(d-2)\right)=0$. Lemma 5 gives $A_{1} \subset D \cup D^{\prime}$. Since $C$ is an irreducible conic containing $A_{1}$, the Bezout theorem gives $b \leq 4$, a contradiction.

(d2) Now assume the existence of a conic $T \subset \mathbb{P}^{2}$ such that $\operatorname{deg}(T \cap(A \cup$ $B)) \geq 2 d+2$. Since $\operatorname{deg}\left(\operatorname{Res}_{T}(A \cup B)\right) \leq d-1$, we have $h^{1}\left(\mathcal{I}_{\operatorname{Res}_{T}(A \cup B)}(d-\right.$ $2))=0$. Hence the case $t=2$ of Lemma 5 gives $A_{1} \subset T$ and $\operatorname{Res}_{T}(A)=$ $B \backslash B \cap T$. Since $b \geq 5, C$ is irreducible and $A_{1} \subset C$, the Bezout theorem gives $T=C$. We get $B \backslash B \cap C=E$. Hence $\sharp(B \cap C) \leq 2 d+1-b$. Hence $\operatorname{deg}(C \cap(A \cup B)) \leq 2 d+1$, a contradiction.

(e) In this step we prove that $\sigma_{s, r}\left(X_{m, d}\right) \neq \emptyset$ for every integer $r$ such that $2 d+3-s \leq r \leq 2 d+s-7$ and $r+s \equiv 1(\bmod 2)$ and hence conclude the proof of Theorem 1. Set $c:=(2 d+3+s-r) / 2$ and $w:=\lfloor c / 2\rfloor$. Since 
$r+s \equiv 1(\bmod 2)$, we have $c \in \mathbb{Z}$. Since $r \geq 2 d+3-s$, we have $c \leq s$. Since $r \leq 2 d+s-7$, we have $c \geq 5$. If $c$ is odd, then we apply Lemma 9. If $c$ is even, then we apply Lemma 8 .

\section{REFERENCES}

[1] E. Ballico and A. Bernardi, Stratification of the fourth secant variety of Veronese variety via the symmetric rank, Adv. Pure Appl. Math., 4 (2) (2013), 215-250; DOI: 10.1515/apam-2013-0015

[2] E. Ballico and A. Bernardi, A partial stratification of secant varieties of Veronese varieties via curvilinear subschemes, arXiv:1010.3546v3, Sarajevo J. Math., 8 (20), (2012), 33-52.

[3] E. Ballico and A. Bernardi, Decomposition of homogeneous polynomials with low rank, Math. Z., 271 (2012), 1141-1149; DOI 10.1007/s00209-011-0907-6.

[4] A. Bernardi and A. Gimigliano, M. Idà, Computing symmetric rank for symmetric tensors, J. Symb. Comput., 46 (2011), 34-55.

[5] A. Bernardi and K. Ranestad, The cactus rank of cubic forms, J. Symb. Comput., 50 (2013) 291-297. DOI: 10.1016/j.jsc.2012.08.001

[6] J. Brachat, P. Comon, B. Mourrain and E. P. Tsigaridas, Symmetric tensor decomposition, Linear Algebra Appl., 433 (11-12) (2010), 1851-1872.

[7] W. Buczyńska and J. Buczyński, Secant varieties to high degree veronese reembeddings, catalecticant matrices and smoothable Gorenstein schemes, arXiv:1012.3562v4 [math.AG], J. Algebr. Geom., (to appear).

[8] J. Buczyński, A. Ginensky and J. M. Landsberg, Determinantal equations for secant varieties and the Eisenbud-Koh-Stillman conjecture, J. London Math. Soc., 88 (2) (2013), 1-24.

[9] G. Comas and M. Seiguer, On the rank of a binary form, Found. Comp. Math., 11 (1) (2011), 65-78.

[10] P. Comon, G. H. Golub, L.-H. Lim, and B. Mourrain, Symmetric tensors and symmetric tensor rank, SIAM J. Matrix Anal., 30 (2008), 1254-1279.

[11] P. Comon and B. Mourrain, Decomposition of Quantics in Sums of Powers of Linear Forms, Signal Processing, Elsevier 53, 2, 1996.

[12] A. Couvreur, The dual minimum distance of arbitrary dimensional algebraic-geometric codes, J. Algebra, 350 (1) (2012), 84-107.

[13] $\mathrm{Ph}$. Ellia and Ch. Peskine, Groupes de points de $\mathbf{P}^{2}$ : caractère et position uniforme, Algebr. geom., (L'Aquila, 1988), 111-116, Lect. Notes Math., 1417, Springer, Berlin, 1990.

[14] J. M. Landsberg, Tensors: Geometry and Applications. Graduate Studies in Mathematics, Vol. 128, Amer. Math. Soc., Providence, 2012.

[15] J. M. Landsberg and Z. Teitler, On the ranks and border ranks of symmetric tensors, Found. Comput. Math., 10, (3)(2010) 339-366.

[16] L.-H. Lim and V. De Silva, Tensor rank and the ill-posedness of the best low-rank approximation problem, SIAM J. Matrix Anal., 30 (3) (2008), 1084-1127.

(Received: October 31, 2012)

(Revised: April 11, 2013)
Department of Mathematics University of Trento 38123 Povo (TN) Italy edoardo.ballico@unitn.it 